\title{
Modeling Framework For Regional Integrated Simulation Of Transportation Network And Activity-Based Demand (Polaris)
}

\author{
Joshua Auld ${ }^{\mathrm{a}}$ \\ Michael Hope ${ }^{\mathrm{a}}$ \\ Hubert Ley ${ }^{\mathrm{a}}$ \\ Bo $\mathrm{Xu}^{\mathrm{a}}$ \\ Kuilin Zhang ${ }^{\mathrm{a}}$ \\ Vadim Sokolov ${ }^{\mathrm{a}^{*}}$
}

\begin{abstract}
Travel demand, traffic flow, network operations and land-use models are typically modeled in a decoupled way, i.e. each of the components is modeled separately assuming the others are fixed. Moreover, the models are often developed by different groups for different contexts, requirement, etc. In this paper we present a prototype of a software framework which allows the user to develop an integrated simulation of a transportation system in a standardized, extensible manner, as well as an implementation of an agentbased planning and network operations micro simulation model using this approach. The project uses an agent-based modeling approach to developing an integrated transportation system model. This allows the model to overcome some of the limitations of traditional aggregated transportation models, particularly with respect to sensitivity to behavioral aspects of the travelers. POLARIS project, then, is intended to develop such an modeling framework and demonstrate the benefits of this approach through the implementation of an integrated travel demand and network operations agent-based micro simulation model. The model is intended to be used in evaluating network operations improvements and I TS implementations from a planning perspective. This paper provides background on the POLARIS modeling framework and details the development of several modules using the framework which form the basis of the planning and operations simulation model.
\end{abstract}

Key words: Integrates transportation simulation; Agent-based; Modeling framework; ITS infrastructure; Traffic operations.

\section{Introduction}

Since the initial development and deployment of Intelligent Transportation Systems (ITS) there has been a need to evaluate the impacts of ITS investment on the transportation system, as

\footnotetext{
${ }^{a}$ Argonne National Laboratory, Argonne, IL, 60439, USA

*Corresponding author: Vadim Sokolov vsokolov@anl.gov http://dx.doi.org/10.14453/isngi2013.proc.43
} 
for any other system investment, which has often been done using simplified sketch planning tools that rely on existing macro demand model, such as IDAS ${ }^{1}$, or a Cube-based ITS tool developed for Florida DOT ${ }^{2}$. Also, USDOT developed the integrated corridor management analysis, modeling, and simulation (ICM AMS) methodologies that assume combining macroscopic travel demand model with meso/macroscopic traffic flow simulation to test impact of different ITS technologies ${ }^{3}$. Another approach used is to combine existing general purpose modeling tools to evaluate limited aspects of the system ${ }^{4-6}$. Dynamic traffic assignment is often the core methodology for both off-line evaluation of intelligent traffic operational planning and on-line traffic control and management of the Intelligent Transportation Systems. Peeta and Ziliaskopoulos ${ }^{7}$ provide an excellent survey of the DTA models in terms of analytical and simulation-based approaches, and point out that the simulation-based DTA models have gained greater acceptability in the context of real-world deployment.

\section{Activity-Based Travel Demand Model}

Travel demand is estimated in POLARIS by using an activity-based demand model. The demand model is agent based, meaning that the individual agent actions and behaviors are simulated. The demand behaviors modeled include time-dependent activity generation, activity attribute planning and replanning, and a detailed activity scheduling model, which resolves schedule conflicts and maintains a consistent daily schedule for the agent. The demand components, including activity generation and activity planning, are also responsive to network and traffic management events, which can result in agent replanning. The demand components implemented in the POLARIS derive from previous work in modeling activity-planning and scheduling behaviors found in the development of the ADAPTS (Agent-based Dynamic Activity Planning and Travel Scheduling) model $^{8}$. We have reorganized the ADAPTS model components to more closely fit the agent-based approach and have implemented them by using the POLARIS modeling language and framework. The ADAPTS model was originally developed to simulate the underlying activity and travel planning and scheduling processes that lead to observed activity-travel patterns. The model was designed to continuously integrate directly with traffic simulation, forming a fully dynamic model, with planning and scheduling occurring in a timedependent manner and impacted by the results of the time-dependent traffic network. The model allows for the simulation of a wide range of travel-demand management policies, especially those which impact the planning process.

The ADAPTS model components have been implemented as a set of agents and sub-agents that perform events that generally represent a single model or process in the original ABM. In the demand model there are two primary executable agent types and several sub-agents. The primary agents are the person agent and the activity-planner agent, which represents the person agent's cognition of the activity planning process. The person agent has a variety of sub-agents, which represent various faculties of the individual. These include the perception agent; the planning agent that generates activities and handles planning or re-planning; the scheduling agent; the movement agent for interacting with the network model; and the routing agent, which interacts with the network model for making routing decisions. The demand model interacts with the network model components through the person agent, by way of the movement handler agent and through the routing faculty. The agent schedules when these actions occur and executes the movement or routing methods of the network model. Similarly, the person interacts with the ITS model components through the perception agent, which updates the person's understanding of 
the network state based on the ITS action. The interactions between the various agents are shown in Figure 1.

The figure shows the primary connections between the agents as described above, the basic simulation process that each agent follows, and the time resolution at which the various discrete events are scheduled. The figure shows that most person agent events occur at regular recurring intervals, currently set to five minutes. The agent polls itself every five minutes to see whether any new activities need to be generated or any activity-travel plans need to be executed, etc. Many of the remaining agent events occur at a near continuous time resolution, however. Activity planning events such as the choice of activity location, mode, etc., as well as the scheduling of the activities, occur throughout the simulation, as do the routing and traveling.

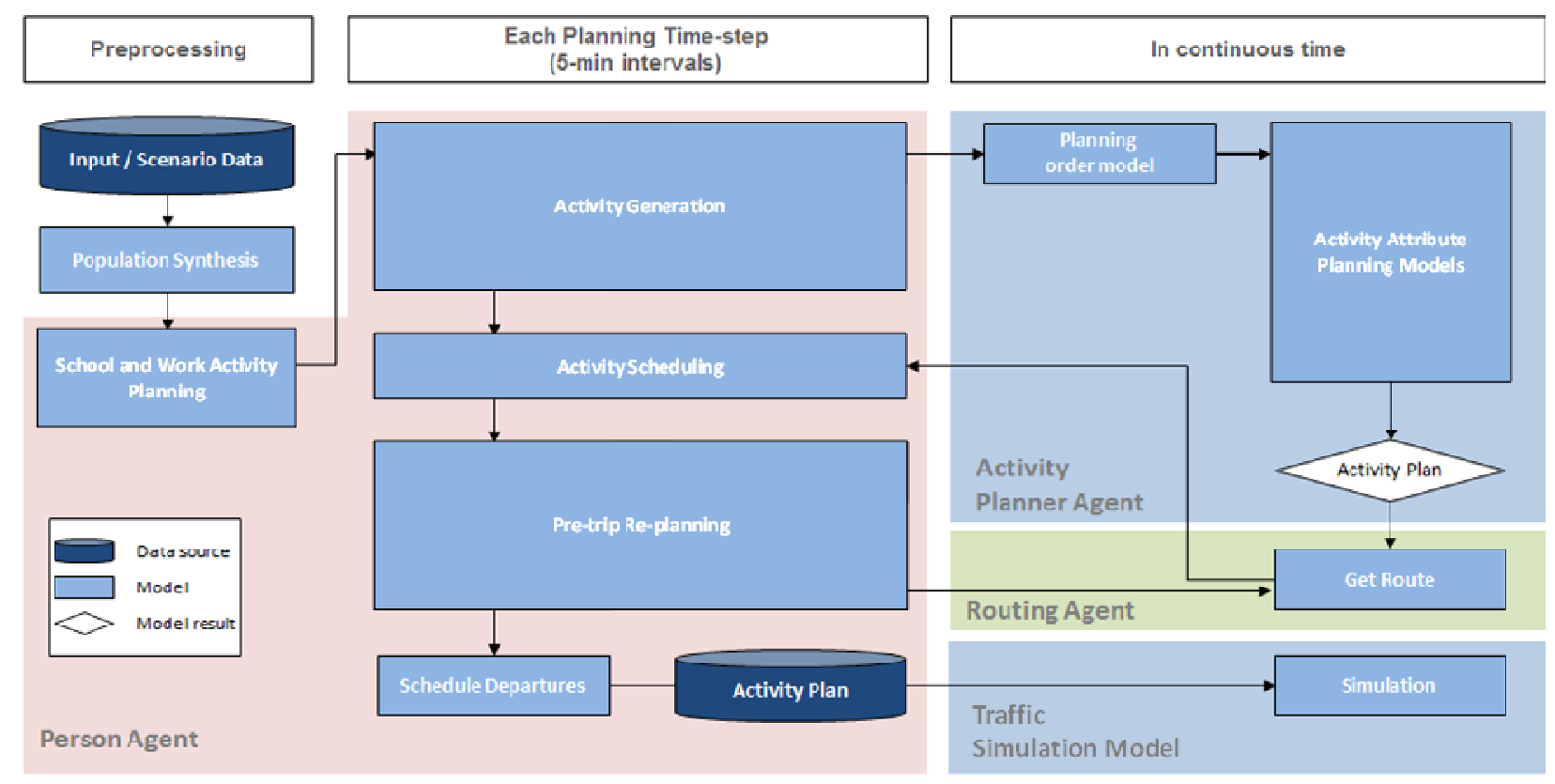

Figure 1.Demand model agent simulation framework

\section{Simulation-Based Dynamic Traffic Assignment Model}

The network model component in POLARIS is a one-shot simulation-based dynamic traffic assignment model that includes the following: (1) a route choice model, (2) a route generation model, and (3) a mesoscopic traffic simulation model. Each of these models is implemented as a collection of POLARIS agents and utilizes the discrete event engine to coordinate execution scheduling and run efficiently. We use two types of route choice model for one-shot DTA, one is route choice based on pre-trip prevailing traffic information; the other is a bounded rationality based en-route switching model for modeling travelers adjust their route using real-time traffic information. We have a future plan to incorporate the DUE approach for an iterative DTA model. 
The agents related to the network model are seamlessly integrated with the demand model agents by providing traffic information for travelers taking demand side actions. The ITS model agents are incorporated by publishing sensor information to the TMC for making traffic operations and control decisions. In turn, the demand side actions and TMC's operations and control decisions also impact the traveler's route choice and traffic flow pattern.

In POLARIS, the network model includes three categories of agents: traveler agents, routing agents, and traffic simulation agents. In the route choice model, routing aspect of the traveler agent is modeled, the route choice decisions are made with respect to its own user characteristics in response to pre-trip and/or en-route traffic information and events from the Internet, variable message sign (VMS), global positioning system (GPS), and radio. A bounded rationality based en-route switching model ${ }^{9-11}$ is implemented by the routing agent. The routing agent is used to calculate the least time routes for individual traveler agents. This calculation employs simulated travel costs. The traffic simulation is conducted by three agents, which represent the overall network, the link, and the intersection. Together these agents perform the task of simulating each individual traveler agent based on the simplified kinematic wave theory of traffic flow ${ }^{12}$. A notable implementation of Newell's model is in an open source dynamic traffic assignment tool DTALite $^{13}$. In addition, an optional intersection control agent simulates signal controls such as pre-timed signals, actuated signals, stop signs, and yield signs. The traffic simulation model also captures dynamic capacity reductions due to events such as weather and accidents.

The interactions among these network model agents, as well as the demand model agents and the ITS model agents, are depicted in Figure 2. Given the inputs of activity plans determined by the demand model agent, each traveler makes route choice decisions on the basis of routes generated by the routing agent using the traveler's route choice behavior with response to information provided by the ITS model agent. These traveler agents with route choice decisions are then simulated by the traffic simulation agents. The output of the traffic simulation model is the network performance and traffic flow pattern, which will be inputs to the route choice model, demand model, and ITS model in POLARIS.

\section{Intelligent Transport Systems, Network Events and Traffic Management Agent}

Currently, the major application of the POLARIS framework is the evaluation of ITS benefits. For that purpose, three components in the POLARIS were developed on top of the demand and network components: network events, ITS infrastructure, and traffic manager agent (TMA).

The ITS infrastructure is an input to the model. Currently, the following types of ITS components are supported: variable message sign (VMS), highway advisory radio (HAR), tow truck dispatch, open hard shoulder sign, and in-road sensor. The in-road sensor is used to model the locations for which the speed and volume data are available to the manager, either from loop detectors or from data vendors. The sensor model allows to estimate the value of available information and to determine how it affects the effectiveness of the management decisions. All the sensors can be used to model noisy data; a user can specify the standard deviation of the measurement error for each of the sensors. Each HAR and VMS can display/broadcast a single message associated with a network event. 
Figure 2.Network model agent simulation framework

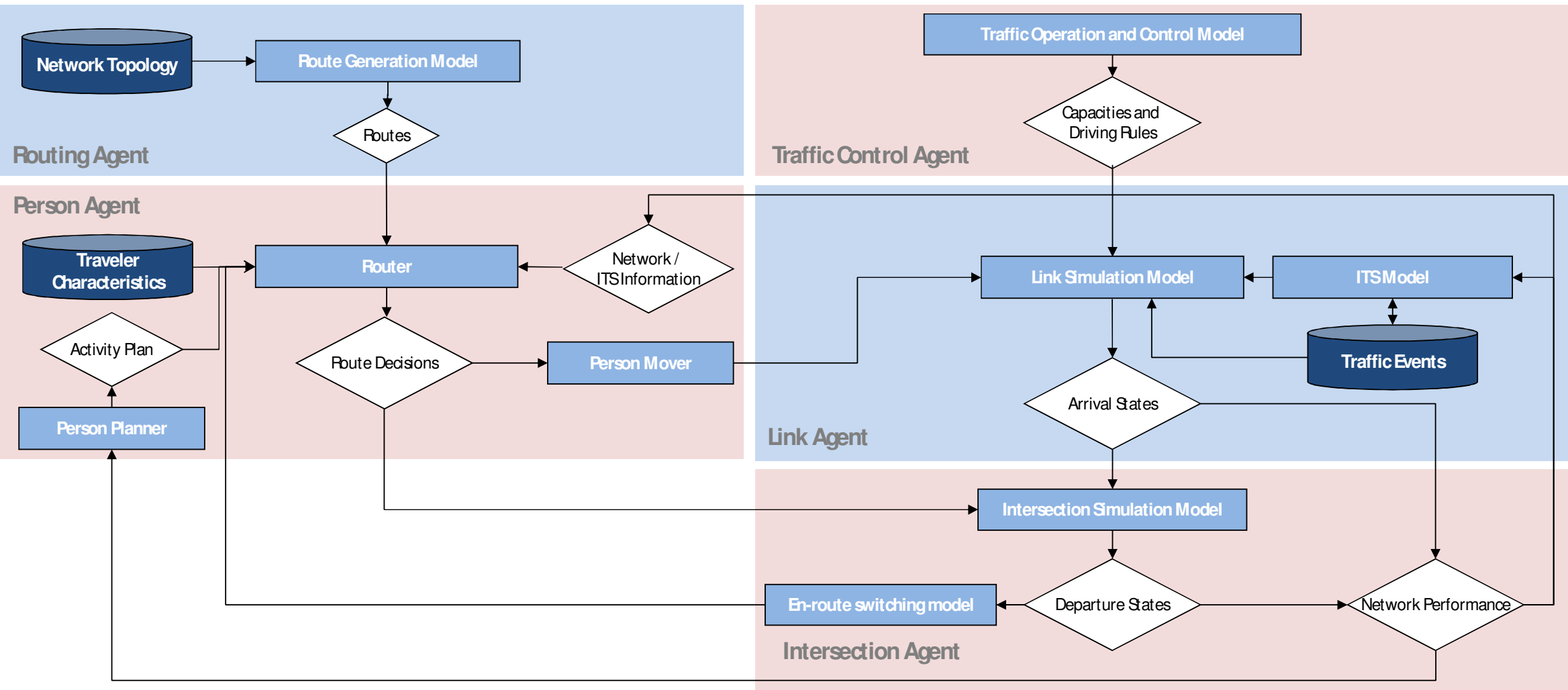


Five types of network events can be modeled by the network events manager: weather, accidents, congestion, lane closure, and travel time. Each event has spatial information about the area affected, start/end time, and event-specific attributes. For example, for a weather event the user can specify type (snow, rain, or fog), level of precipitation, visibility, asphalt conditions, etc. IBM's Smarter Cities incident representation semantics was used to model most of the network events in POLARIS.

The role of the traffic manager can be played either by a human or by a robot. Both of the approaches serve a different purpose. The human in the loop setup allows interaction with the ongoing simulation and hand-crafting the ITS response to a given scenario. This approach may be able to be used effectively for educational purposes or as a simulator-trainer for traffic engineers.

The performance of the transportation system and the benefits of ITS and traffic management need to be evaluated under many different assumptions. Treating weather, special events, road construction, and time of day as random variables leads to a large number of possible scenarios that need to be evaluated. It is error prone and requires significant amount of time to hand craft ITS response strategies to all possible scenarios. Thus, as a part of the framework an automated traffic manager agent is being developed. The goal of this agent is to monitor the status of the transportation network (speed, travel times, etc.) as well as network-related events (weather, incidents, etc.) and decide on a response that would mitigate unusual congestion levels on the network. This aspect of the model is intended to allow planning agencies to analyze the benefits of different network operational improvements. Currently, the traffic manager has two roles. The first is to identify unusual congestion by observing the network performance from the ongoing simulation and statistically comparing the simulation data with the historical data. The second role is to each highway advisory radio and VMS sign to an event. To do so, the agent calculates a relevance score, which is a function that maps a pair (network event, VMS/HAR, etc.) to a score. The information on the event with the highest score will be displayed or broadcast. To calculate the relevance score, the agent uses statistical data from historical travel patterns (events on heavily traveled parts of the network will score higher) as well as event parameters (the more severe the event, the higher the score).

\section{Running cases}

Another component provided in the base POLARIS framework (i.e., independent of any specific model implementation) is the scenario manager, a set of tools used to define, execute in parallel, and analyze various traffic simulation scenarios in terms of conditions such as weather, demand, incidents, and so on. The scenario manager allows the modeler to account for nontypical conditions (inclement weather, special events, and incidents) in evaluating the model response. Furthermore, it allows for the computation of MOEs based on the expected distribution of simulated scenarios. These features are particularly useful for benefit/cost analysis of ITS or other transportation improvement projects, where a significant amount of the expected benefits is incurred for non-typical conditions. The scenario manager follows the guidance provided in "Operations Benefit/Cost Analysis Desk Reference"" and "Guidebook on the Analysis of Active Transportation and Demand Management Strategies"15 for scenario generation and analysis. In addition, it is able to exploit high performance computing resources, such as TRACC's 92 node 
Zephyr cluster, to simulate multiple scenarios concurrently. The major components of the scenario manager are summarized below:

- Scenario generator - generates multiple scenarios based on a JavaScript Object Notation format scenario description. The scenario description defines scenarios using parameters such as weather conditions and incident types as well as the occurrence probability associated with each scenario.

- Scenario executor - executes scenarios as computing jobs in a computer cluster. The computer cluster automatically schedules and assigns the jobs so that they are executed on different computers in parallel. The scenario manager supports the PBS (portable batch system) job management system and works with Linux cluster environments.

- Scenario monitor - monitors the status of each submitted job, including the progress percentage, estimated remaining running time, summary of network statistics, and so on.

- Scenario analyzer - collects the performance measures reported for each scenario, assigns weights to them by their occurrence probabilities, and sums across scenarios to provide overall measures of effectiveness. The scenario analyzer also produces plots of performance measures from the outputs of scenario execution for comparative analysis.

The scenario manager provides two modes for generating weather/incident scenarios, namely, the comprehensive mode and the representative mode. These two modes differ in whether the duration of the weather/incident event covers the entire simulated period and where the adjustments of capacity and free-flow speed are made. In the comprehensive mode, the duration of an event covers the entire simulated period, and adjustments are made to the link input. In the representative mode, an event occurs during a sub-period of the simulated period, and adjustments are made online by the network simulator. Each of the two modes has its advantages and usages. The advantage of the comprehensive mode is that the network simulator does not need to know how to model weather/incident events; the modeling knowledge is outside the network simulator, provided by the scenario manager. Thus, the comprehensive mode can be used by existing network simulators other than POLARIS. The advantage of the representative mode is that it can manage scenarios in which there are multiple events and each event has a temporary time scope. Therefore, the representative mode is suitable for scenarios with incidents.

Initial tests were run using the network simulation model to route and simulate 27 million trips in the Chicago metropolitan network over a 24 hour period. These tests were performed on one node of TRACC's Zephyr cluster, the system specification is as follows: two AMD 6273 2.3GHZ CPUs, 64G RAM, CentOS/Linux 6.2. The wall time for a multi-thread case is approximately 75 minutes.

\section{Conclusion}

This paper has detailed the development of a modeling framework and demonstrates the benefits of this approach through the implementation of an integrated travel-demand and network operations agent-based model. This model (Integrated ABM Simulator) is intended to be used in evaluating network operations improvements and ITS implementations from a planning perspective. Details have been provided about the POLARIS modeling framework and several 
ancillary tools used for model development, as well as a description of the Integrated ABM Simulator model developed using POLARIS.

\section{References}

${ }^{1}$ McHale, G., "IDAS (Its Deployment Analysis System): A Tool For Integrating Its Into The Planning Process", Public Roads, Vol. 63, No. 6, 2000, pp. 11-13.

${ }^{2}$ Hadi, M., Xiao, Y., Ozen, H., and Alvarez, P. Evaluation Tools to Support ITS Planning Process: Development of a Sketch Planning Tool in FSUTMS/Cube Environment, 2008.

${ }^{3}$ Alexiadis, V., "Integrated Corridor Management Analysis, Modeling and Simulation (AMS) Methodology", No FHWA-JPO-08-034. Tech. rept. FHWA, 2008.

${ }^{4}$ Cragg, C. A., and Demetsky, M. J., "Simulation Analysis of Route Diversion Strategies for Freeway Incident Management.", Tech. rept., 1995.

${ }^{5}$ Barcelo, J., Garcá, D., and Kirschfink, H., "Integration of AIMSUN NG and ALMO: A software platform to assist advanced traffic management decisions", Proceedings of the 11th World Conference on ITS, Nagoya, 2004.

${ }^{6}$ Tettamanti, T., Varga, I., Kulcsár, B., and Bokor, J., "Model predictive control in urban traffic network management", 16th IEEE Mediterranean Conference on Control and Automation, 2008, pp 1538-1543.

${ }^{7}$ Peeta, S., and Ziliaskopoulos, A. K., "Foundations of dynamic traffic assignment: The past, the present and the future", Networks and Spatial Economics, Vol. 1, No. 3-4, 2001, pp. 233265. http://dx.doi.org/10.1023/A:1012827724856

${ }^{8}$ Auld, J., and Mohammadian, A., "Framework for the development of the Agent-based Dynamic Activity Planning and Travel Scheduling (ADAPTS) model", Transportation Letters, International Journal of Transportation Research, Vol. 1, No. 3, 2009, pp. 245-255. http://dx.doi.org/10.3328/TL.2009.01.03.245-255

${ }^{9}$ Mahmassani, H. S., and Stephan, D. G., Experimental investigation of route and departure time choice dynamics of urban commuters, 1988.

${ }^{10}$ Mahmassani, H. S., and Jayakrishnan, R., "System performance and user response under realtime information in a congested traffic corridor", Transportation Research Part A: General, Vol. 25, No. 5, 1991, pp. 293-307. http://dx.doi.org/10.1016/0191-2607(91)90145-G

${ }^{11}$ Jayakrishnan, R., Mahmassani, H. S., and Hu, T.Y., "An evaluation tool for advanced traffic information and management systems in urban networks", Transportation Research Part C: Emerging Technologies, Vol. 2, No. 3, 1994, pp. 129-147. http://dx.doi.org/10.1016/0968090X(94)90005-1

${ }^{12}$ Newell, G. F., "A simplified theory of kinematic waves in highway traffic, Part I: General theory", Transportation Research Part B: Methodological, Vol. 27, No. 4, 1993, pp. 281287. http://dx.doi.org/10.1016/0191-2615(93)90038-C

${ }^{13}$ Zhou, X., Lu, C.C., and Zhang, K., "Dynamic Origin-Destination Demand Flow Estimation Under Congested Traffic Conditions: A General Framework", Transportation Research Board 91st Annual Meeting, 2012.

${ }^{14}$ FHWA., Operations Benefit/Cost Analysis Desk Reference. US Department of Transportation. Federal Highway Administration. Tech. rept. FHWA, 2012, URL: http://www.ops.fhwa.dot.gov/publications/fhwahop12028/fhwahop12028.pdf

${ }^{15}$ FHWA., Guidebook on the Analysis of Active Transportation and Demand Management Strategies. Tech. rept. FHWA, 2013. 